\title{
A coarse-grained field theory for density fluctuations and correlation functions of galaxies and clusters
}

\author{
Y. Zhang \\ Astrophysics Center, University of Science and Technology of China, Hefei, Anhui, PR China \\ e-mail: yzh@ustc.edu.cn
}

Received 25 September 2006 / Accepted 21 November 2006

\begin{abstract}
Aims. We aim to investigate the large-scale structure of the universe from a field-theoretical perspective, and to give a unifying understanding of several seemingly unrelated observed features of clusterings of galaxies and clusters, such as the dependence of clustering amplitude on mass and luminosity, the departure of the correlation function $\xi(r)$ from the power law $\propto\left(r_{0} / r\right)^{1.7}$, the oscillatory behavior of $\xi_{\mathrm{cc}}(r)$ for clusters on very large scales, and the scaling of $r_{0}$ with the mean separation $d$ of clusters.

Methods. We present a coarse-grained field theory of density fluctuations for a Newtonian self-gravitating many-body system, apply it to a homogeneous Universe with small density fluctuations, and uniformly treat the clustering of all types of galactic objects in terms of the field of density fluctuations.

Results. The Jeans length $\lambda_{0}$, a unique physical scale for a gravitating system, appears naturally as the characteristic scale underlying the large-scale structure. Under a Gaussian approximation the analytic expressions of $\xi(r)$ and $P(k)$ are obtained. The correlation amplitude is proportional to the galactic mass, $\xi(r) \propto m$, and $\xi(r)$ departures from the power law $\left(r_{0} / r\right)^{1.7}$ around $r \sim 20 h^{-1} \mathrm{Mpc}$, and is oscillating over large scales $\sim 100 h^{-1} \mathrm{Mpc}$ and damped to zero. The spectrum amplitude is inversely proportional to the galactic number density, $P(k) \propto 1 / n$, and $P(k) \propto k^{-2.2}$ in the range $k \simeq(0.05 \sim 0.3) h \mathrm{Mpc}^{-1}$. The scaling $r_{0} \propto d^{0.3 \sim 0.5}$ holds quantitatively. Conclusions. These preliminary analytic results under the Gaussian approximation already qualitatively explain these pronounced features of the observed large-scale structures. Yet on small scales the predicted clustering is insufficient, and the peak of $P(k)$ is too sharp. These deficiencies are due to neglecting higher order nonlinear effects, which need to be studied further.
\end{abstract}

Key words. cosmology: large-scale structure of Universe - cosmology: theory - galaxies: clusters: general - gravitation hydrodynamics - instabilities

\section{Introduction}

In past decades, great progress has been made in understanding the large-scale structure of the universe. Not only have observations of the major galaxy surveys, such as SDSS, 2dF, APM, etc., (Tegmark 2004; Zehavi 2005; Colless 2001; Hawkins 2003; Madgwick 2003; Percival 2005; Maddox 1996; Padilla 2003) revealed cosmic structures of increasingly large sky dimension, but theoretical studies have also achieved important results through both analytical modeling and numerical simulations. The correlation functions of galactic objects are key physical quantities, which contain important information, both dynamic and statistical, about the cosmic structure. Observations have shown that the correlation functions of various galactic objects, such as galaxies, groups of galaxies, quasars, and clusters, are usually fitted by a single canonical power law $\xi(r)=\left(r_{0} / r\right)^{\gamma}$ in the range $r \lesssim 4 r_{0}$ with the index $\gamma \sim 1.7$ and the "correlation scale" $r_{0} \sim 5 h^{-1} \mathrm{Mpc}$ for galaxies, $\sim 10 h^{-1} \mathrm{Mpc}$ for galaxy groups, and $\sim 20 h^{-1} \mathrm{Mpc}$ for clusters, respectively (Peebles 1980, 1993; Bahcall et al. 2003). Also, $r_{0}$ increases with mass, richness, and luminosity of galactic objects. Moreover, $r_{0}$ scales with the mean cluster separation $d$ as $r_{0}(d) \propto d^{0.3 \sim 0.5}$ (Croft 1997; Gonzalez 2002; Bahcall et al. 2003; Zandivarez et al. 2003). On large scales $\xi(r)$ departures from the power law, drops steeply to zero, and has an oscillating behavior on very large scales $\geq 100 h^{-1}$ Mpc. These observed features have been thought to be a theoretical challenge (Bahcall 1996). From the point of view of dynamics, the Universe filled with various galactic objects is a many-body system with an attractive gravity between bodies. A powerful, systematic approach to dealing with statistical dynamics of this kind of system is through introducing the coarse-graining order parameter, which converts the degrees of freedom of discrete particles into a continuous field. Therefore, a field theory arises, which combines both the statistical and dynamical properties of the system. The method has been very successful in revealing the correlation properties for a variety of systems near phase transition (Zinn-Justin 1996). Therefore, it should be appropriate to employ the method to study the density fluctuations of the homogeneous universe as a self-gravitating systems. In this paper we will formulate such a field theory of density fluctuations and apply it to the Universe on large scales. It turns out that our analytic result at the level of Gaussian approximation already qualitatively interprets the several above-mentioned features simultaneously. Different from the conventional analytical studies, such as the perturbation theory (Bernardeau 2001) and the thermodynamic formulation (Saslaw 2000), our approach is a field-theoretical study, like those used in condensed matter and in particle physics, the differential equation of the 2-point correlation function can be derived explicitly. 


\section{Field equation of correlation function}

Consider a system of $N$ galaxies of mass $m$ in the Universe with the Hamiltonian $H=\sum_{i}^{N} p_{i}^{2} / 2 m-\sum_{i<j}^{N} G m^{2} /\left|\boldsymbol{r}_{i}-\boldsymbol{r}_{j}\right|$. The grand partition function of this system at temperature $T$ is

$Z=\sum_{N=0}^{\infty} \frac{z^{N}}{N !} \int \prod_{i=1}^{N} \frac{\mathrm{d}^{3} p_{i} \mathrm{~d}^{3} r_{i}}{(2 \pi)^{3}} \mathrm{e}^{-H / T}$,

where $z$ is the fugacity. By using the Hubbard-Stratonovich transformation (Zinn-Justin 1996), it can be cast into a path integral: $Z=\int D \phi \exp \left[-\beta^{-1} \int \mathrm{d}^{3} \boldsymbol{r} \mathcal{L}(\phi)\right]$, where $\phi$ is the gravitational field, $\beta \equiv 4 \pi G m / c_{\mathrm{s}}^{2}$ of dimension $m^{-1}, c_{\mathrm{s}} \equiv(T / m)^{1 / 2}$ the sound speed, the effective Lagrangian is $\mathcal{L}(\phi)=\frac{1}{2}(\nabla \phi)^{2}-$ $k_{J}^{2} \mathrm{e}^{\phi}$, and $k_{J} \equiv\left(4 \pi G \rho_{0} / c_{\mathrm{s}}^{2}\right)^{1 / 2}$ is the Jeans wavenumber. The term $-k_{J}^{2} \mathrm{e}^{\phi}$ has a minus sign because gravity is attractive. By the Poisson's equation $\nabla^{2} \phi(\boldsymbol{r})+k_{J}^{2} \mathrm{e}^{\phi}=0$, the mass density $\rho$ is related to the $\phi$ field by $\rho(\boldsymbol{r})=m n(\boldsymbol{r})=\rho_{0} \mathrm{e}^{\phi(\boldsymbol{r})}$. So $\rho_{0}$ is the constant mass density when $\phi=0$. We define a dimensionless re-scaled mass density field

$\psi(\boldsymbol{r}) \equiv \mathrm{e}^{\phi(\boldsymbol{r})}=\rho(\boldsymbol{r}) / \rho_{0}$,

viewed as the coarse-grained order parameter, and introduce a Schwinger type of external source $J$ coupled with $\psi$ in the effective Lagrangian

$\mathcal{L}(\psi, J)=\frac{1}{2}\left(\frac{\nabla \psi}{\psi}\right)^{2}-k_{J}^{2} \psi-J \psi$.

The source $J$ is used to handle the functional derivatives with ease. The equation for $\psi$ in the presence of $J$ is

$\nabla^{2} \psi-\frac{1}{\psi}(\nabla \psi)^{2}+k_{J}^{2} \psi^{2}+J \psi^{2}=0$,

the generating functional is

$Z[J]=\int D \psi \exp \left[-\beta^{-1} \int \mathrm{d}^{3} \boldsymbol{r} \mathcal{L}(\psi, J)\right]$,

and the $n$-point connected correlation function is

$$
\begin{aligned}
G_{\mathrm{c}}^{(n)}\left(\boldsymbol{r}_{1}, \ldots, \boldsymbol{r}_{n}\right) & =\left\langle\delta \psi\left(\boldsymbol{r}_{1}\right) \ldots \delta \psi\left(\boldsymbol{r}_{n}\right)\right\rangle \\
& =\left.\beta^{n} \frac{\delta^{n} \ln Z[J]}{\delta J\left(\boldsymbol{r}_{1}\right) \ldots \delta J\left(\boldsymbol{r}_{n}\right)}\right|_{J=0},
\end{aligned}
$$

where $\delta \psi(\boldsymbol{r})=\psi(\boldsymbol{r})-\langle\psi(\boldsymbol{r})\rangle$ is the fluctuation field around the expectation value $\langle\psi(\boldsymbol{r})\rangle$. The standard way to obtain the equation for the 2-point correlation function $G_{\mathrm{c}}^{(2)}(\boldsymbol{r})$ is the following (Goldenfeld 1992). Taking the expected value of Eq. (4) in the presence of $J$; using the approximation $\left\langle(\nabla \psi)^{2} / \psi\right\rangle_{J} \simeq$ $\left(\nabla\langle\psi\rangle_{J}\right)^{2} /\langle\psi\rangle_{J}$, which is equivalent to neglecting high-order terms of $\delta \psi$; taking the functional derivative of this equation w.r.t. the source $J$, and setting $J=0$, we end up with

$$
\begin{array}{r}
\nabla^{2} G_{\mathrm{c}}^{(2)}(\boldsymbol{r})+\left(\frac{\nabla\langle\psi\rangle}{\langle\psi\rangle}\right)^{2} G_{\mathrm{c}}^{(2)}(\boldsymbol{r})-\frac{2}{\langle\psi\rangle} \nabla\langle\psi\rangle \cdot \nabla G_{\mathrm{c}}^{(2)}(\boldsymbol{r}) \\
+2 k_{J}^{2}\langle\psi\rangle G_{\mathrm{c}}^{(2)}(\boldsymbol{r})=-\langle\psi\rangle^{2} \beta \delta^{(3)}(\boldsymbol{r}),
\end{array}
$$

where $G_{\mathrm{c}}^{(2)}\left(\boldsymbol{r}-\boldsymbol{r}^{\prime}\right)=\beta \delta\langle\psi(\boldsymbol{r})\rangle_{J=0} / \delta J\left(\boldsymbol{r}^{\prime}\right)$ has been used, and $\langle\psi\rangle \equiv\langle\psi\rangle_{J=0}$ is the background field depending on the position $\boldsymbol{r}$ in general. We remark that when higher order terms in $1 / \psi \simeq 1 / \psi_{0}+\delta \psi / \psi_{0}^{2}+\ldots$ were kept, one would end up with a nonlinear equation that was more complicated than Eq. (7).
Now assume the homogeneity of the Universe on a large scale. Then the background density field is constant $\langle\psi\rangle=\psi_{0}=1$, and Eq. (7) reduces to the Helmholtz equation with a point source:

$\nabla^{2} \xi(\boldsymbol{r})+2 k_{J}^{2} \xi(\boldsymbol{r})=-\frac{4 \pi G m}{c_{\mathrm{s}}^{2}} \delta^{(3)}(\boldsymbol{r})$

where we have used a simple notation $\xi(\boldsymbol{r}) \equiv G_{\mathrm{c}}^{(2)}(\boldsymbol{r})$. The approximation level of Eq. (8) is essentially that of the Gaussian model in the Landau-Ginzburg theory. The solution is the Green's function

$\xi(r)=A\left(c \frac{\cos \left(k_{0} r\right)}{r}+(1-c) \frac{\sin \left(k_{0} r\right)}{r}\right)$,

where the amplitude $A \equiv G m / c_{\mathrm{s}}^{2}$, the parameter $c$ determines the contributions from independent cos and sin modes, and the characteristic wavenumber $k_{0} \equiv \sqrt{2} k_{J}$. Thus the wavelength $\lambda_{0}=\lambda_{J} / \sqrt{2}$ occurs as the characteristic scale that underlies the large-scale structure, where $\lambda_{J}$ is the Jeans length. The "correlation scale" $r_{0}$ in the power law is only empiric not fundamental. The amplitude is $A \propto G m / c_{\mathrm{s}}^{2} r_{0}$, the ratio of the gravitational correlation energy $G m^{2} / r_{0}$ to twice the kinetic energy $m c_{\mathrm{s}}^{2}$ of peculiar motion of galaxies. Thus, a greater gravitational energy yields a larger correlation amplitude, similar to the conclusion by Saslaw (2000). A comparison with the Landau-Ginzburg theory will be suggestive. Recall that for a fluid, with the dominant force between molecules being repulsive, the Gaussian approximation yields a correlation function $\propto \mathrm{e}^{-r / \eta} / r$, where $\eta$ is the correlation length of phase transition. Contrarily, Eq. (9) is oscillating, instead of decaying, because gravity is attractive. In this sense, the self-gravitating system is always at the critical point of "phase transition" with $\eta \rightarrow \infty$. Therefore, it is pertinent to study the density fluctuations of the homogeneous universe within the framework of the field theory, where the coarse-grained order parameter is the rescaled density field $\psi$. If the gravity were repulsive, the term $2 k_{J}^{2} \psi$ in Eq. (8) would have a minus sign, and Eq. (9) would be of the same form $\xi(r) \propto \mathrm{e}^{-\left|k_{0}\right| r} / r$ as for a fluid. Using the definition of the projected correlation function (Davis \& Peebles 1983) $w_{\mathrm{p}}\left(r_{\mathrm{p}}\right)=2 \int_{r_{\mathrm{p}}}^{\infty} \xi(r)\left(r^{2}-r_{\mathrm{p}}^{2}\right)^{-1 / 2} r \mathrm{~d} r$, and Eq. (9), one gets $w_{\mathrm{p}}\left(r_{\mathrm{p}}\right)=\pi A\left[-c N_{0}\left(k_{0} r_{\mathrm{p}}\right)+(1-c) J_{0}\left(k_{0} r_{\mathrm{p}}\right)\right]$, where $N_{0}$ is the Neumann function and $J_{0}$ the Bessel function. Therefore, the amplitude is also proportional to the mass, $w_{\mathrm{p}} \propto m$, and $w_{\mathrm{p}}\left(r_{\mathrm{p}}\right)$ has a strong departure from the power-law and drops steeply to zero, agreeing with the observations (Budavari 2003; Zehavi et al. 2002, 2005).

\section{Implications}

Now examine some implications of $\xi(r)$ for the large-scale structure. Although, as the Gaussian approximation, Eq. (9) does not yet give a detailed account of the large-scale structure, it already reveals the following pronounced features.

1) Galactic objects in our theory are all treated as gravitating particles, and have the same form of correlation functions as Eq. (9), differing only in $A$ and $k_{0}$. Indeed, the observed $\xi(r)$ for galaxies, groups, and clusters all have a similar shape, for instance, the correlation function of RELEX clusters divided by $\sim 10$ coincide with that of EPS galaxies (Guzzo 2000).

2) Since the amplitude $A \propto m$, galactic objects with a greater mass have a higher correlation. Besides, since richness is proportional to $m$ by definition and more luminous galaxies generally have greater mass, this result naturally explains 


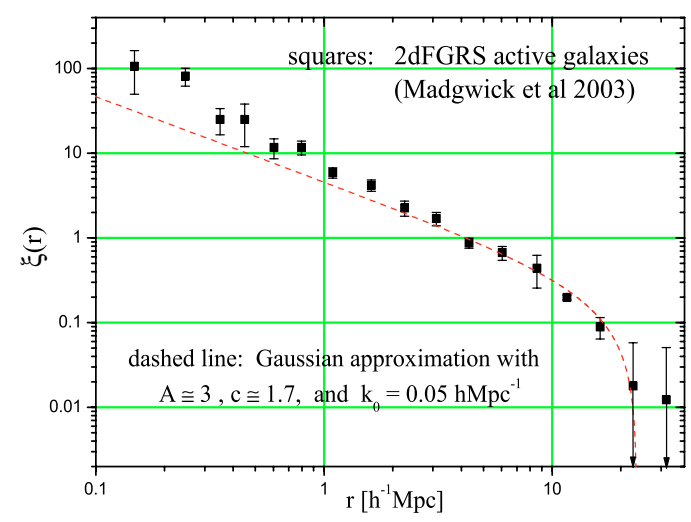

Fig. 1. The calculated $\xi(r)$ in Gaussian approximation compared with the observed $\xi_{\mathrm{gg}}(r)$ of 2dFGRS (Madgwick et al. 2003).

why the correlation amplitudes increase with richness and luminosity of galactic objects without recourse to the bias model. Attempts have been made to explain this in terms of the statistics of rare peak events (Kaiser 1984). Moreover, early-type galaxies (ellipticals) have a mean number density $n$ lower than that of late-type (spirals) galaxies and are more likely located in denser regions, thus they represent a greater mass of the region (Davis \& Geller 1976; Madgwick 2003) and have a higher correlation.

3) $\xi(r)$ has a departure from the canonical power law, as is observed. The plot $\xi(r)$ in Fig. 1 fits with $\xi_{\text {gg }}(r)$ of the 2 df galaxies (Madgwick 2003). The parameters $A \simeq 3 h^{-1} \mathrm{Mpc}$, $c \simeq 1.7$, and $k_{0} \simeq 0.05 h \mathrm{Mpc}^{-1}$ are taken. It is seen that the departure from the power law occurs at $\sim 8 h^{-1} \mathrm{Mpc}$, and $\xi(r)$ goes to zero at $\simeq(20 \sim 30) h^{-1}$ Mpc. But on small scales $<2 h^{-1} \mathrm{Mpc}, \xi(r) \propto r^{-1}$, lower than the observed. This small-scale deficiency of correlation is expected since significant clustering effects of higher order terms of $\delta \psi$ have been neglected in Eq. (8). We expect these nonlinear effects will improve this small-scale correlation. For groups and clusters, fitting can be done similarly, requiring a larger $A$ and a slightly smaller $k_{0}$. However, the redshift-space correlation function from the SDSS luminous red galaxies crosses to zero only after $100 \mathrm{~h}^{-1} \mathrm{Mpc}$ (Eisenstein et al. 2005; Huetsi 2006). It is difficult for our theory, and it might indicate that either the universe is still less relaxed, or the higher order nonlinear effects need to be taken into account.

4) The shape of observed correlation function of galaxies depends on their "color". The red galaxies are more luminous with a slightly higher mean number density $n$, and exhibit a steeper and higher-amplitude correlation function than the blue galaxies (Zehavi 2002, 2005). This is explained by Eq. (9), which, for a larger $k_{0} \propto(\mathrm{nm})^{1 / 2}$, is steeper and has a higher amplitude on small scales.

5) As mentioned at the beginning, the observations show that the "correlation scale" $r_{0}$ increases with the mean separation $d$ in the same way as $r_{0}(d) \propto d^{0.3 \sim 0.5}$ (Bahcall 1996; Bahcall et al. 2003; Croft 1997). The underlying mechanism was thought to be a scaling behavior (Szalay \& Schramm 1985). In our theory, if we adopt the power law fitting $r_{0}(d) \propto$ $\xi^{1 / 1.7}$ with the $\xi$ given in Eq. (9), then, for typical clusters, $r_{0}(d) \sim 20 \cos ^{1 / 1.7}\left[(30 / d)^{3 / 2}\right]$, increasing in the range $d=10 \sim 80 h^{-1} \mathrm{Mpc}$, as shown in Fig. 2. The result is in qualitative agreement with the observation.

6) On very large scales, $\xi(r)$ is a damped oscillation about zero. Some surveys have indicated this feature with

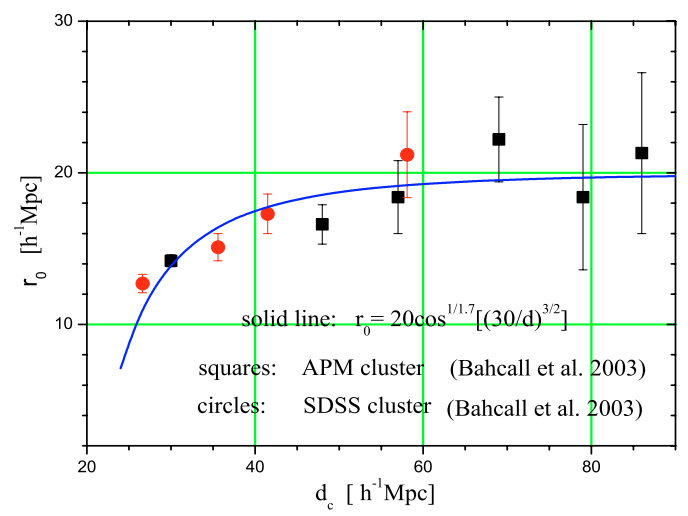

Fig. 2. The calculated scaling $r_{0}(d)$ compared with the observation (Bahcall 1996; Bahcall et al. 2003).

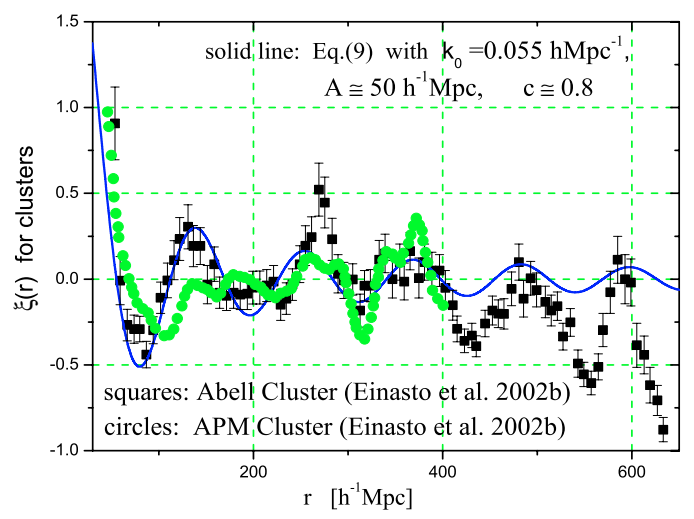

Fig. 3. The large-scale oscillations of $\xi(r)$ compared with the observed $\xi_{\mathrm{cc}}(r)$ of clusters (Einasto 2002; Einasto et al. 2002).

$\lambda_{0} \simeq 120 \sim 200 h^{-1} \mathrm{Mpc}$ (Broadhurst et al. 1990; Tucker 1997; Einasto et al. 1997a, 1997b; Einasto 2002; Einasto et al. 2002; Tago 2002; Yanata 2005). Figure 3 gives a fit to the observed $\xi_{\text {cc }}(r)$ of clusters, requiring $A \simeq 50 h^{-1} \mathrm{Mpc}$ and $k_{0} \simeq 0.05 h \mathrm{Mpc}^{-1}$. Thus the Gaussian approximation in Eq. (9) gives a good description of the oscillatory correlation on very large scales.

\section{Power spectrum}

The power spectrum is the Fourier transform of the correlation function, and can be also derived directly by Fourier transforming Eq. (8),

$P(k)=\frac{1}{2 n} \frac{1}{\left(\frac{k}{k_{0}}\right)^{2}-1}$.

Firstly, this predicts that $P(k)$ has a higher amplitude for galactic objects with a smaller spatial number density $n$, agreeing with a whole variety of surveys. For instance, the observed $P(k)$ for all Abell clusters is lower than that for Abell clusters with measured redshifts, and the same fact is also found for APM clusters (Einasto 2002b). Secondly, $P(k)$ has a sharp peak at $k=k_{0}$, corresponding to the periodic wavelength $\lambda_{0}=2 \pi / k_{0}$. Taking the observed value $\lambda_{0} \simeq 120 \sim 200 h^{-1}$ Mpc yields the peak location $k_{0} \simeq 0.031 \sim 0.052 \mathrm{~h} \mathrm{Mpc}^{-1}$. The peak has been, to some degree, indicated by several cluster surveys (Ratcliffe et al. 1996; Tadros et al. 1998; Hoyle et al. 1999; Collins 2000; Schuecker et al. 2001; Carretti et al. 2002; Einasto 2002; Einasto et al. 2002; Tago et al. 2002; Padilla \& Baugh 2003; 


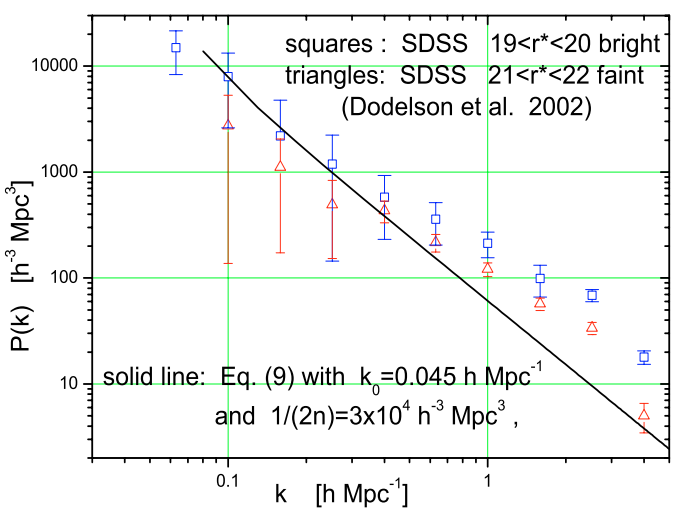

Fig. 4. The analytic $P(k)$ in Eq. (10) compared with the SDSS galaxies (Dodelson et al. 2002) in the range $k \simeq(0.1 \sim 5) h \mathrm{Mpc}^{-1}$.

Percival 2005). Thirdly, as shown in Fig. 4, for large scales $k \simeq(0.05 \sim 0.3) h \mathrm{Mpc}^{-1}$, (10) gives $P(k) \propto k^{-2.2}$, agreeing well with the major surveys (Peacock 1997, 1999; Carretti et al. 2002; Dodelson et al. 2002), whereas the power law with $\gamma \sim 1.7$ would yield $\propto k^{-1.3}$. However, for small scales $k \simeq(0.4 \sim$ 3) $h \mathrm{Mpc}^{-1}$, Eq. (10) gives $P(k) \propto k^{-2}$, the observed is $\propto k^{-1.2}$ (Peacock 1999; Dodelson et al. 2003). This small-scale deficiency is the same phenomenon occurring in $\xi(r)$ noticed before, also due to neglect of the nonlinear terms. But there are two more difficulties for $P(k)$ in Eq. (10). It takes on negative values on large scales with $k<k_{0}$, and its peak is too sharp. We expect that, when the nonlinear and frictional effects are properly taken into account, the negative power at $k<k_{0}$ may also be alleviated and the peak may be smoothed to certain extent. These are obviously different from the observed power spectrum. A real spectrum cannot have an infinitely sharp peak, which should be smoothed by some damping effects. Recent observations have indicated that the measured $P(k)$ has a rather flat peak with large systematic errors on large scales (Outram et al. 2003; Padilla \& Baugh 2003; Tegmark et al. 2004; Maller et al. 2005; Percival 2005).

\section{Conclusion and discussion}

The universe containing galaxies or clusters is a many-particle system and can be studied with the help of field theoretical techniques. In this regard, the coarse-grained density field is of use, giving a perspective other than those well-known methods, such as simulations, perturbation, thermodynamics, etc. The expansion of the universe and the evolution have not been included in this paper. We study the asymptotic relaxed state of a Newtonian self-gravitating system with random velocities. This is valid for large-scale structures, where the cosmic time scale $1 / H_{0}$ is longer than the local crossing time scale. Our theory here yields the Jeans scale $k_{0} \simeq(0.04 \sim 0.06) h \mathrm{Mpc}^{-1}$ as the unique scale underlying the large-scale structure. Even at the level of the Gaussian approximation, the theory already simultaneously interprets several important features of the observed large-scale structures, although some of them have been thought to be puzzling and seemingly unrelated. But the results give insufficient clustering on small scales. So it is necessary to take into account higher order terms of $\delta \psi$ neglected in Eq. (8). This may enhance the small-scale clustering and smooth out the sharp peak of the power spectrum.

Acknowledgements. This work was supported by the CNSF No. 10173008, NKBRSF G19990754, SRFDP, and CAS.

\section{References}

Bahcall, N. A. 1996, in Unsolved Problems in Astrophysics, ed. J. P. Bahcall, \& J. P. Ostriker (New Jersey: Princeton Univ. Press)

Bahcall, N. A., Dong, F., Hao, L., et al. 2003, ApJ, 599, 814

Bernardeau, F., Colombi, S., Gonzanaga, E., \& Sccoccimarro, R. 2002, Phys. Rep., 367,1

Broadhurst, T. J., Ellis, R. S., Koo, D. C., \& Szalay, A. S. 1990, Nature, 343, 726 Budavari, T., Connolly, A. J., Szalay, A. S., et al. 2003, ApJ, 595, 59

Carretti, E., Bertoni, C., Messina, A., Zucca, E., \& Guzzo, E. 2001, MNRAS, 324,1029

Chavanis, P. H. 2006, Phyisica A, 361, 55

Colless, M., Dalton, G., Maddox, S., et al. 2001, MNRAS, 328, 1039

Collins, C. A., Guzzo, L., Böhringer, H., et al. 2000, MNRAS, 319, 939

Croft, R. A. C., Dalton, G. B., Efstathiou, G., Sutherland, W. J., \& Maddox, S. J. 1997, MNRAS, 291, 305

Davis, M., \& Geller, M. J. 1976, ApJ, 208, 13

Davis, M., \& Peebles, P. J. E. 1983, ApJ, 267, 465

Dodelson, S., Narayanan, V. K., Tegmark, M., et al. 2002, ApJ, 572, 140

Einasto, J. 2002, in The Ninth Marcel Grossmann Meeting. Proceedings of the MGIXMM Meeting held at The University of Rome, ed. V. G. Gurzadyan, R. T. Jantzen, \& R. Ruffini (Singapore: World Scientific Publishing)

Einasto, J., Einasto, M., Gottloeber, S., et al. 1997a, Nature, 385, 139

Einasto, J., Einasto, M., Frisch, P., et al. 1997b, MNRAS, 289, 801

Einasto, M., Einasto, J., Tago, E., et al. 2002, AJ, 123, 51

Eisenstein, D., Zehavi, I., Hogg, D. W., et al. 2005, ApJ, 633, 560

Goldenfeld, N. 1992, Lectures on Phase Transitions and the Renormalization Group, Addison-Wesley

Gonzalez, A. H., Zaritsky, D., \& Wechler, R. H. 2002, ApJ, 571, 129

Guzzo, L., Bartlett, J. G., Cappi, A., et al. 2000, A\&A, 355, 1

Hawkins, E., Maddox, S., Cole, S., et al. 2003, MNRAS, 346, 78

Hoyle, F., Baugh, C. M., Shanks, T., \& Ratcliffe, A. 1999, MNRAS, 309, 659

Huetsi, G. 2006, A\&A, 449, 891

Kaiser, N. 1984, ApJ, 284, L9

Maddox, S. J., Efstathiou, G., \& Sutherland, W. J. 1996, MNRAS, 238, 1227

Madgwick, D. S., Hawkins, E., Lahav, O., et al. 2003, MNRAS, 344, 847

Maller, A. H., McIntosh, D. H., Katz, N., \& Weinberg, M. D. 2005, ApJ, 619, 147

Outram, P. J., Hoyle, F., Shanks, T., et al. 2003, MNRAS, 342, 483

Padilla, N. D., \& Baugh, C. M. 2003, MNRAS, 343, 796

Peacock, J. A. 1997, MNRAS, 284, 885

Peacock, J. A. 1999, Cosmological Physics (Cambridge: Cambridge Univ. Press) Peebles, P. J. E. 1980, The Large Scale Structure of the Universe (New Jersey: Princeton Univ. Press)

Peebles, P. J. E. 1993, Principles of Physical Cosmology (New Jersey: Princeton Univ. Press)

Percival, W. J. 2005, MNRAS, 356, 1169

Ratcliffe, A., Shanks, T., Broadbent, A., et al. 1996, MNRAS, 281, L47

Saslaw, W. C. 2000, The Distribution of Galaxies (Cambridge: Cambridge Univ. Press)

Schuecker, P., et al. 2001, A\&A, 386, 86

Szalay, A., \& Schramm, D. N. 1985, Nature, 314, 718

Tadros, H., Efstathiou, G., \& Dalton, G. 1998, MNRAS, 296, 995

Tago, E., Saar, E., Einasto, J., et al. 2002, AJ, 123, 37

Tegmark, M., Blanton, M. R., Strauss, M. A., et al. 2004, ApJ, 606, 702

Tucker, D. L., Oemler, A., Jr., Kirshner, R. P., et al. 1997, MNRAS, 285, L5

Yahata, K., Suto, Y., Kayo, I., et al. 2005, PASJ, 57, 529

Zandivarez, A., Merchan, M. E., \& Padilla, N. D. 2003, MNRAS, 344, 247

Zehavi, I., Blanton, M. R., Frieman, J. A., et al. 2002, ApJ, 571, 172

Zehavi, I., Zheng, Z., Weinberg, D. H., et al. 2005, ApJ, 630, 1

Zinn-Justin, J. 1996, Quantum Field Theory and Critical Phenomena (Oxford: Oxford Univ. Press) 